\title{
Evaluating the Lifetime Performance Index Based on the Bayesian Estimation for the Rayleigh Lifetime Products with the Upper Record Values
}

\author{
Wen-Chuan Lee, ${ }^{1}$ Jong-Wuu Wu, ${ }^{2}$ Ching-Wen Hong, ${ }^{3}$ and Shie-Fan Hong ${ }^{2}$ \\ ${ }^{1}$ Department of International Business, Chang Jung Christian University, Tainan 71101, Taiwan \\ ${ }^{2}$ Department of Applied Mathematics, National Chiayi University, 300 Syuefu Road, Chiayi City 60004, Taiwan \\ ${ }^{3}$ Department of Information Management, Shih Chien University, Kaohsiung Campus, Kaohsiung 84550, Taiwan \\ Correspondence should be addressed to Jong-Wuu Wu; jwwu@mail.ncyu.edu.tw
}

Received 20 October 2012; Accepted 17 December 2012

Academic Editor: Chong Lin

Copyright (c) 2013 Wen-Chuan Lee et al. This is an open access article distributed under the Creative Commons Attribution License, which permits unrestricted use, distribution, and reproduction in any medium, provided the original work is properly cited.

\begin{abstract}
Quality management is very important for many manufacturing industries. Process capability analysis has been widely applied in the field of quality control to monitor the performance of industrial processes. Hence, the lifetime performance index $C_{L}$ is utilized to measure the performance of product, where $L$ is the lower specification limit. This study constructs a Bayesian estimator of $C_{L}$ under a Rayleigh distribution with the upper record values. The Bayesian estimations are based on squared-error loss function, linear exponential loss function, and general entropy loss function, respectively. Further, the Bayesian estimators of $C_{L}$ are utilized to construct the testing procedure for $C_{L}$ based on a credible interval in the condition of known $L$. The proposed testing procedure not only can handle nonnormal lifetime data, but also can handle the upper record values. Moreover, the managers can employ the testing procedure to determine whether the lifetime performance of the Rayleigh products adheres to the required level. The hypothesis testing procedure is a quality performance assessment system in enterprise resource planning (ERP).
\end{abstract}

\section{Introduction}

Process capability analysis is an effective means to measure the performance and potential capabilities of a process. Process capability indices (PCIs) are utilized to assess whether product quality meets the required level in manufacturing industries. For instance, the lifetime of electronic components exhibits a larger-the-better type of quality characteristic. Montgomery [1] proposed the process capability index $C_{L}$ to evaluate the lifetime performance of electronic components, where $L$ is the lower specification limit. Tong et al. [2] constructed the uniformly minimum variance unbiased estimator (UMVUE) of $C_{L}$ and proposed a hypothesis testing procedure for the complete sample from a one-parameter exponential distribution. In addition, the lifetime performance index $C_{L}$ also is applied to evaluate the lifetime of product in the censored sample. For instance, Hong et al. [3, 4] constructed the lifetime performance index $C_{L}$ to evaluate business performance under the right type II censored sample and proposed a confidence interval for Pareto's distribution. Wu et al. [5] proposed a hypothesis testing procedure based on a maximum likelihood estimator (MLE) of $C_{L}$ to evaluate the product quality for two-parameter exponential distribution under the right type II censored sample. Lee et al. [6] also proposed a hypothesis testing procedure based on a MLE of $C_{L}$ to evaluate product quality for exponential distribution under the progressively type II right censored sample. Lee et al. [7] also constructed an UMVUE of $C_{L}$ and developed a testing procedure for the performance index of products with the exponential distribution based on the type II right censored sample. All of the above $C_{L}$ have been utilized to evaluate the quality performance for complete data and censored data. Nevertheless, record values often arise in industrial stress testing and other similar situations.

Record values and the associated statistics are of interest and important in many real-life applications. In industry and reliability studies, many products fail under stress. For example, a battery dies under the stress of time. But the precise 
breaking stress or failure point varies even among identical items. Hence, in such experiments, measurements may be made sequentially and only the record values (lower or upper) are observed. Record values arise naturally in many reallife applications involving data relating to weather, sports, economics, and life tests. According to the model of Chandler [8], there are some situations in lifetime testing experiments where the failure time of a product is recorded if it exceeds all preceding failure times. These recorded failure times are the upper record value sequence. In general, let $X_{1}, X_{2}, \ldots$ be a sequence of independent and identically distributed (i.i.d.) random variables having the same distribution as the (population) random variable $X$ with the cumulative distribution function (c.d.f) $F(x)$ and the probability density function (p.d.f) $f(x)$. An observation $X_{j}$ will be called an upper record value if it exceeds in value all of the preceding observations, that is, if $X_{j}>X_{i}$, for every $i<j$. The sequence of record times $T_{n}, n \geq 1$ is defined as follows.

Let $T_{1}=1$ with probability 1 , for $n \geq 2, T_{n}=\min \{j$ : $\left.X_{j}>X_{T_{n-1}}\right\}$. A sequence of upper record values $\left\{X_{U(n)}\right\}$ is then defined by

$$
X_{U(n)}=X_{T_{n}}, \quad n=1,2, \ldots
$$

Let $\underset{\sim}{X}=\left\{X_{U(1)}, X_{U(2)}, \ldots, X_{U(n)}\right\}$ be the first $n$ upper record values arising from a sequence of i.i.d. random variables $X_{1}, X_{2}, \ldots$ with c.d.f. $F(x)$.

In this study, we consider the case of the upper record values instead of complete data or censored data. In order to evaluate the lifetime performance of nonnormal data with upper record values, the lifetime performance index $C_{L}$ is utilized to measure product quality under the Rayleigh distribution with the upper record values. The Rayleigh distribution is a nonnormal distribution and a special case of the Weibull distribution, which provides a population model useful in several areas of statistics, including life testing and reliability whose age with time as its failure rate is a linear function of time. Bhattacharya and Tyagi [9] mentioned that in some clinical studies dealing with cancer patients the survival pattern follows the Rayleigh distribution. Cliff and Ord [10] showed that the Rayleigh distribution arises as the distribution of the distance between an individual and its nearest neighbor when the special pattern is generated by the Poisson process. Dyer and Whisenand [11] demonstrated the importance of this distribution in communication engineering (also see Soliman and Al-Aboud [12]). The p.d.f. and c.d.f. of the Rayleigh distribution are given, respectively, by

$$
\begin{aligned}
& f(x)=\frac{x}{\theta^{2}} e^{-x^{2} / 2 \theta^{2}}, \\
& F(x)=1-e^{-x^{2} / 2 \theta^{2}},
\end{aligned}
$$

where $x>0$ and $\theta>0$, respectively. When $X$ comes from the Rayleigh distribution, the mean of $X$ is $E(X)=\sqrt{\pi / 2} \theta$, and the standard deviation of $X$ is $\sigma=\sqrt{(4-\pi) / 2} \theta$.

Bayesian and non-Bayesian approaches have been used to obtain the estimators of the parameter $\theta$ under the Rayleigh distribution with the upper record values. Soliman and AlAboud [12] compared the performance of the Bayesian estimators with non-Bayesian estimators such as the MLE and the best linear unbiased (BLUE) estimator. The Bayesian estimators are developed under symmetric and nonsymmetric loss functions. The symmetric loss function is squared-error (SE) loss function. The nonsymmetric loss function includes linear exponential (LINEX) and general entropy (GE) loss functions. In recent decades, the Bayesian viewpoint has received frequent attention for analyzing failure data and other time-to-event data and has been often proposed as a valid alternative to traditional statistical perspectives. The Bayesian approach to estimation of the parameters and reliability analysis allows prior subjective knowledge on lifetime parameters and technical information on the failure mechanism as well as experimental data. Bayesian methods usually require less sample data to achieve the same quality of inferences than methods based on sampling theory (also see [12]).

The main aim of this study will construct the Bayesian estimator of $C_{L}$ under a Rayleigh distribution with upper record values. The Bayesian estimators of $C_{L}$ are developed under symmetric and nonsymmetric loss functions. The estimators of $C_{L}$ are then utilized to develop a credible interval, respectively. The new testing procedures of credible interval for $C_{L}$ can be employed by managers to assess whether the products performance adheres to the required level in the condition of known $L$. The new proposed testing procedures can handle nonnormal lifetime data with upper record values. Moreover, we will evaluate the performance of the new proposed testing procedures with Bayesian and nonBayesian approaches.

The rest of this study is organized as follows. Section 2 introduces some properties of the lifetime performance index for lifetime of product with the Rayleigh distribution. Section 3 discusses the relationship between the lifetime performance index and conforming rate. Section 4 develops a hypothesis testing procedure for the lifetime performance index $C_{L}$ with the non-Bayesian approach. Section 5 develops a hypothesis testing procedure for the lifetime performance index $C_{L}$ with the Bayesian approach. Section 6 discusses the Monte Carlo simulation algorithm of confidence (or credible) level. One numerical example and concluding remarks are made in Sections 7 and 8, respectively.

\section{The Lifetime Performance Index}

Montgomery [1] has developed a process capability index $C_{L}$ to measure the larger-the-better quality characteristic. Then, $C_{L}$ is defined by

$$
C_{L}=\frac{\mu-L}{\sigma},
$$

where $\mu, \sigma$, and $L$ are the process mean, the process standard deviation, and the lower specification limit, respectively.

To assess the product performance of products, $C_{L}$ can be defined as the lifetime performance index. If $X$ comes from the Rayleigh distribution, then the lifetime performance 
index $C_{L}$ can be rewritten as

$$
\begin{array}{r}
C_{L}=\frac{\mu-L}{\sigma}=\frac{\sqrt{\pi / 2} \theta-L}{\sqrt{(4-\pi) / 2} \theta}= \\
-\sqrt{\frac{\pi}{4-\pi}}-\sqrt{\frac{2}{4-\pi}} \frac{L}{\theta}, \\
-\infty<C_{L}<\sqrt{\frac{\pi}{4-\pi}},
\end{array}
$$

where $\mu=E(X)=\sqrt{\pi / 2} \theta$ is the process mean, $\sigma=$ $\sqrt{\operatorname{Var}(X)}=\sqrt{(4-\pi) / 2} \theta$ is the process standard deviation, and $L$ is the lower specification limit.

\section{The Conforming Rate}

If the lifetime of a product $X$ exceeds the lower specification limit $L$, then the product is defined as a conforming product. The ratio of conforming products is known as the conforming rate and can be defined as

$$
\begin{aligned}
P_{r}=P(X \geq L) & =e^{-(1 / 2)\left(\sqrt{\pi / 2}-\sqrt{(4-\pi) / 2} C_{L}\right)^{2}}, \\
& -\infty<C_{L}<\sqrt{\pi /(4-\pi)} .
\end{aligned}
$$

Obviously, a strictly increasing relationship exists between the conforming rate $P_{r}$ and the lifetime performance index $C_{L}$. Table 1 lists various $C_{L}$ values and the corresponding conforming rate $P_{r}$ by using STATISTICA software [13] (also see [14]).

For the $C_{L}$ values which are not listed in Table 1, the conforming rate $P_{r}$ can be obtained through interpolation. In addition, since a one-to-one mathematical relationship exists between the conforming rate $P_{r}$ and the lifetime performance index $C_{L}$. Therefore, utilizing the one-to-one relationship between $P_{r}$ and $C_{L}$, lifetime performance index can be a flexible and effective tool, not only evaluating product performance, but also estimating the conforming rate $P_{r}$.

\section{Testing Procedure for the Lifetime Performance Index $C_{L}$ with Non-Bayesian Approach}

This section will apply non-Bayesian approach to construct a maximum likelihood estimator (MLE) of $C_{L}$ under a Rayleigh distribution with upper record values. The MLE of $C_{L}$ is then utilized to develop a new hypothesis testing procedure in the condition of known $L$. Assuming that the required index value of lifetime performance is larger than $c_{0}$, where $c_{0}$ denotes the target value, the null hypothesis $H_{0}: C_{L} \leq c_{0}$ and the alternative hypothesis $H_{1}: C_{L}>\mathcal{c}_{0}$ are constructed. Based on the new hypothesis testing procedure, the lifetime performance of products is easy to assess.

Let $X$ be the lifetime of such a product, and $X$ has a Rayleigh distribution with the p.d.f. as given by (2). Let $\underset{\sim}{X}=\left(X_{U(1)}, X_{U(2)}, \ldots, X_{U(n)}\right)$ be the first $n$ upper record values arising from a sequence of i.i.d. Rayleigh variables with p.d.f. as given by (2). Since the joint p.d.f. of $\left(X_{U(1)}, X_{U(2)}, \ldots, X_{U(n)}\right)$ is

$$
f\left(x_{U(n)}\right) \prod_{i=1}^{n-1} \frac{f\left(x_{U(i)}\right)}{1-F\left(x_{U(i)}\right)},
$$

where $f(x)$ and $F(x)$ are the p.d.f. and c.d.f. of $X$, respectively (also see $[12,15,16]$ ). So, the likelihood function of $\left(X_{U(1)}, X_{U(2)}, \ldots, X_{U(n)}\right)$ is given as

$$
L\left(\theta \mid x_{U(1)}, \ldots, x_{U(n)}\right)=\left\{\prod_{i=1}^{n} \frac{x_{U(i)}}{\theta^{2}}\right\} \exp \left(-\frac{x_{U(n)}^{2}}{2 \theta^{2}}\right) .
$$

The natural logarithm of the likelihood function with (8) is

$$
\ln L\left(\theta \mid x_{U(1)}, \ldots, x_{U(n)}\right)=\sum_{i=1}^{n} \ln \left(x_{U(i)}\right)-2 n \ln \theta-\frac{x_{U(n)}^{2}}{2 \theta^{2}} .
$$

Upon differentiating (9) with respect to $\theta$ and equating the result to zero, the MLE of the parameter $\theta$ can be shown to be

$$
\widehat{\theta}_{\mathrm{MLE}}=\frac{X_{U(n)}}{\sqrt{2 n}}
$$

(also see [12]). By using the invariance of MLE (see Zehna [17]), the MLE of $C_{L}$ can be written as given by

$$
\begin{aligned}
\widehat{C}_{L, \mathrm{MLE}} & =\sqrt{\frac{\pi}{4-\pi}}-\sqrt{\frac{2}{4-\pi}} \frac{L}{\widehat{\hat{\theta}}_{\mathrm{MLE}}} \\
& =\sqrt{\frac{\pi}{4-\pi}}-\sqrt{\frac{2}{4-\pi}} \cdot L \cdot \frac{\sqrt{2 n}}{X_{U(n)}} .
\end{aligned}
$$

Construct a statistical testing procedure to assess whether the lifetime performance index adheres to the required level. The one-sided confidence interval for $C_{L}$ is obtained using the pivotal quantity $X_{U(n)}^{2} / \theta^{2}$. By using the pivotal quantity $X_{U(n)}^{2} / \theta^{2}$, given the specified significance level $\alpha$, the level $100(1-\alpha) \%$ one-sided confidence interval for $C_{L}$ can be derived as follows.

Since the pivotal quantity $X_{U(n)}^{2} / \theta^{2} \sim \chi_{2 n}^{2}$, and $\chi_{2 n, 1-\alpha}^{2}$ function which represents the lower $1-\alpha$ percentile of $\chi_{2 n}^{2}$, then

$$
\begin{gathered}
P\left(\frac{X_{U(n)}^{2}}{\theta^{2}} \leq \chi_{2 n, 1-\alpha}^{2}\right)=1-\alpha, \\
\Longrightarrow P\left(C_{L} \geq \sqrt{\frac{\pi}{4-\pi}}-\left(\sqrt{\frac{\pi}{4-\pi}}-\widehat{C}_{L, \mathrm{MLE}}\right)\left(\frac{\chi_{2 n, 1-\alpha}^{2}}{2 n}\right)^{1 / 2}\right) \\
=1-\alpha,
\end{gathered}
$$

where $C_{L}=\sqrt{\pi /(4-\pi)}-\sqrt{2 /(4-\pi)}(L / \theta)$. From (12), we 
TABLE 1: The lifetime performance index $C_{L}$ versus the conforming rate $P_{r}$.

\begin{tabular}{|c|c|c|c|c|c|}
\hline$C_{L}$ & $P_{r}$ & $C_{L}$ & $P_{r}$ & $C_{L}$ & $P_{r}$ \\
\hline$-\infty$ & 0.000000 & 0.40 & 0.611832 & 1.20 & 0.896627 \\
\hline-4.50 & 0.000147 & 0.45 & 0.631685 & 1.25 & 0.909965 \\
\hline-4.00 & 0.000551 & 0.50 & 0.651484 & 1.30 & 0.922511 \\
\hline-3.50 & 0.001858 & 0.55 & 0.671182 & 1.35 & 0.934227 \\
\hline-3.00 & 0.005628 & 0.60 & 0.690734 & 1.40 & 0.945076 \\
\hline-2.50 & 0.015308 & 0.65 & 0.710094 & 1.45 & 0.955027 \\
\hline-2.00 & 0.037404 & 0.70 & 0.729213 & 1.50 & 0.964047 \\
\hline-1.50 & 0.082094 & 0.75 & 0.748044 & 1.55 & 0.972109 \\
\hline-1.00 & 0.161849 & 0.80 & 0.766539 & 1.60 & 0.979187 \\
\hline-0.50 & 0.286621 & 0.85 & 0.784648 & 1.65 & 0.985259 \\
\hline 0.00 & 0.455938 & 0.90 & 0.802324 & 1.70 & 0.990306 \\
\hline 0.15 & 0.513214 & 0.95 & 0.819518 & 1.75 & 0.994310 \\
\hline 0.20 & 0.532718 & 1.00 & 0.836183 & 1.80 & 0.997261 \\
\hline 0.25 & 0.552370 & 1.05 & 0.852271 & 1.85 & 0.999147 \\
\hline 0.30 & 0.572132 & 1.10 & 0.867738 & 1.90 & 0.999963 \\
\hline 0.35 & 0.591967 & 1.15 & 0.882538 & 1.91 & 0.999997 \\
\hline
\end{tabular}

obtain that a $100(1-\alpha) \%$ one-sided confidence interval for $C_{L}$ is

$$
C_{L} \geq \sqrt{\frac{\pi}{4-\pi}}-\left(\sqrt{\frac{\pi}{4-\pi}}-\widehat{C}_{L, \mathrm{MLE}}\right)\left(\frac{\chi_{2 n, 1-\alpha}^{2}}{2 n}\right)^{1 / 2}
$$

where $\widehat{C}_{L, M L E}$ is given by (11). Therefore, the $100(1-\alpha) \%$ lower confidence interval bound for $C_{L}$ can be written as

$$
\underline{\mathrm{LB}}_{\mathrm{MLE}}=\sqrt{\frac{\pi}{4-\pi}}-\left(\sqrt{\frac{\pi}{4-\pi}}-\widehat{C}_{L, \mathrm{MLE}}\right)\left(\frac{\chi_{2 n, 1-\alpha}^{2}}{2 n}\right)^{1 / 2},
$$

where $\widehat{C}_{L, M L E}, \alpha$, and $n$ denote the MLE of $C_{L}$, the specified significance level, and the upper record sample of size, respectively.

The managers can use the one-sided confidence interval to determine whether the product performance adheres to the required level. The proposed testing procedure of $C_{L}$ with $\widehat{C}_{L, M L E}$ can be organized as follows.

Step 1. Determine the lower lifetime limit $L$ for products and the performance index value $c_{0}$, then the testing null hypothesis $H_{0}: C_{L} \leq c_{0}$ and the alternative hypothesis $H_{1}: C_{L}>c_{0}$ are constructed.

Step 2. Specify a significance level $\alpha$.

Step 3. Given the upper record values $\underset{\sim}{x}=\left(x_{U(1)}, x_{U(2)}, \ldots\right.$, $\left.x_{U(n)}\right)$, the lower lifetime limit $L$, and the significance level $\alpha$, then we can calculate the $100(1-\alpha) \%$ one-sided confidence interval $\left[\underline{\mathrm{LB}}_{\mathrm{MLE}}, \infty\right)$ for $C_{L}$, where $\underline{\mathrm{LB}}_{\mathrm{MLE}}$ as the definition of (14).

Step 4. The decision rule of statistical test is provided as follows:
(1) if the performance index value $c_{0} \notin\left[\underline{\mathrm{LB}}_{\mathrm{MLE}}, \infty\right)$, then we will reject $H_{0}$. It is concluded that the lifetime performance index of products meets the required level;

(2) if the performance index value $c_{0} \in\left[\underline{\mathrm{LB}}_{\mathrm{MLE}}, \infty\right)$, then we do not reject $H_{0}$. It is concluded that the lifetime performance index of products does not meet the required level.

\section{Testing Procedure for the Lifetime Performance Index $C_{L}$ with the Bayesian Approach}

This section will apply the Bayesian approach to construct an estimator of $C_{L}$ under a Rayleigh distribution with upper record values. The Bayesian estimators are developed under symmetric and nonsymmetric loss functions. The symmetric loss function is squared-error (SE) loss function. The nonsymmetric loss function includes linear exponential (LINEX) and general entropy (GE) loss functions. The Bayesian estimator of $C_{L}$ is then utilized to develop a new hypothesis testing procedure in the condition of known L. Assuming that the required index value of lifetime performance is larger than $c_{0}$, where $c_{0}$ denotes the target value, the null hypothesis $H_{0}: C_{L} \leq c_{0}$ and the alternative hypothesis $H_{1}: C_{L}>c_{0}$ are constructed. Based on the new hypothesis testing procedure, the lifetime performance of products is easy to assess.

5.1. Testing Procedure for the Lifetime Performance Index $C_{L}$ with the Bayesian Estimator under Squared-Error Loss Function. Let $X$ be the lifetime of such a product, and $X$ has a Rayleigh distribution with the p.d.f. as given by (2). We consider the conjugate prior distribution of the form which is defined by the square-root inverted-gamma density 
as follows:

$$
\pi_{1}(\theta)=\frac{a^{b}}{\Gamma(b) 2^{b-1}} \theta^{-2 b-1} e^{-a / 2 \theta^{2}},
$$

where $\theta>0, a>0$, and $b>0$.

$$
\begin{gathered}
E(\theta)=\left(\frac{a}{2}\right)^{1 / 2} \frac{\Gamma(b-1 / 2)}{\Gamma(b)} \\
\operatorname{Var}(\theta)=\frac{a}{2(b-1)}-\left(\frac{a}{2}\right)\left[\frac{\Gamma(b-1 / 2)}{\Gamma(b)}\right]^{2}, \quad b>1 .
\end{gathered}
$$

With record values, $n$ products (or items) take place on test. Consider $\left(X_{U(1)}, X_{U(2)}, \ldots, X_{U(n)}\right)$ is the upper record values. We can obtain that the posterior p.d.f. of $\theta \mid\left(X_{U(1)}\right.$, $\left.X_{U(2)}, \ldots, X_{U(n)}\right)$ is given as

$$
\begin{aligned}
p_{1}\left(\theta \mid x_{U(1)}, \ldots, x_{U(n)}\right) & \\
= & \frac{L\left(\theta \mid x_{U(1)}, \ldots, x_{U(n)}\right) \pi_{1}(\theta)}{\int_{0}^{\infty} L\left(\theta \mid x_{U(1)}, \ldots, x_{U(n)}\right) \pi_{1}(\theta) d \theta} \\
& =\frac{\theta^{-2(n+b)-1}\left(x_{U(n)}^{2}+a\right)^{(n+b)} e^{-\left(x_{U(n)}+a\right) / 2 \theta^{2}}}{\Gamma(n+b) 2^{n+b-1}},
\end{aligned}
$$

where $\theta>0$, and the likelihood function $L\left(\theta \mid x_{U(1)}, \ldots\right.$, $\left.x_{U(n)}\right)$ as (8). as

Let $t=x_{U(n)}^{2}+a$, then the posterior p.d.f. can be rewritten

$$
p_{1}\left(\theta \mid x_{U(1)}, \ldots, x_{U(n)}\right)=\frac{\theta^{-2(n+b)-1} t^{(n+b)} e^{-t / 2 \theta^{2}}}{\Gamma(n+b) 2^{n+b-1}},
$$

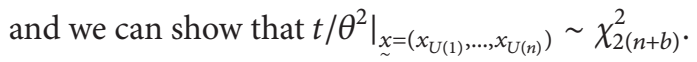

The Bayesian estimator $\widehat{\theta}_{\mathrm{BS}}$ of $\theta$ based on the squared error (SE) loss function $L\left(\theta^{*}, \theta\right)=\left(\theta^{*}-\theta\right)^{2}$ can be derived as follows:

$$
\begin{aligned}
E(\theta \mid & \left.x_{U(1)}, \ldots, x_{U(n)}\right) \\
& =\int_{0}^{\infty} \theta p_{1}\left(\theta \mid x_{U(1)}, \ldots, x_{U(n)}\right) d \theta \\
& =\frac{\Gamma(n+b-1 / 2)}{\Gamma(n+b)}\left(\frac{t}{2}\right)^{1 / 2},
\end{aligned}
$$

where $t=x_{U(n)}^{2}+a, n+b-1 / 2>0$.

Hence, the Bayesian estimator of $\theta$ based on SE loss function is given by

$$
\widehat{\theta}_{\mathrm{BS}}=\frac{\Gamma(n+b-1 / 2)}{\Gamma(n+b)}\left(\frac{T}{2}\right)^{1 / 2},
$$

where $T=X_{U(n)}^{2}+a, n+b-1 / 2>0, a$, and $b$ are the parameters of prior distribution with density as (15).

The lifetime performance index $C_{L}$ of Rayleigh products can be written as

$$
C_{L}=\sqrt{\frac{\pi}{4-\pi}}-\sqrt{\frac{2}{4-\pi}} \frac{L}{\theta}, \quad-\infty<C_{L}<\sqrt{\frac{\pi}{4-\pi}} .
$$

By using (5) and the Bayesian estimator $\widehat{\theta}_{\mathrm{BS}}$ as $(20), \widehat{C}_{L, \mathrm{BS}}$ based on the Bayesian estimator $\widehat{\theta}_{\mathrm{BS}}$ of $\theta$ is given by

$$
\begin{aligned}
\widehat{C}_{L, \mathrm{BS}} & =\sqrt{\frac{\pi}{4-\pi}}-\sqrt{\frac{2}{4-\pi}} \frac{L}{\widehat{\theta}_{\mathrm{BS}}} \\
& =\sqrt{\frac{\pi}{4-\pi}}-\sqrt{\frac{2}{4-\pi}} \cdot L \cdot\left[\frac{\Gamma(n+b-1 / 2)}{\Gamma(n+b)}\left(\frac{T}{2}\right)^{1 / 2}\right]^{-1},
\end{aligned}
$$

where $T=X_{U(n)}^{2}+a, n+b-1 / 2>0, a$, and $b$ are the parameters of prior distribution with density as in (15).

We construct a statistical testing procedure to assess whether the lifetime performance index adheres to the required level. The one-sided credible confidence interval for

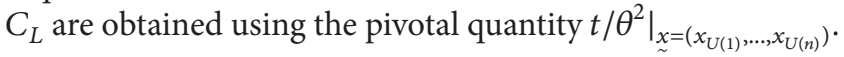
By using the pivotal quantity $t /\left.\theta^{2}\right|_{x=\left(x_{U(1)}, \ldots, x_{U(n)}\right)}$, given the specified significance level $\alpha$, the level $100(1-\alpha) \%$ one-sided credible interval for $C_{L}$ can be derived as follows.

Since the pivotal quantity $t /\left.\theta^{2}\right|_{\sim=\left(x_{U(1)}, \ldots, x_{U(n)}\right)} \sim \chi_{2(n+b)}^{2}$, and $\chi_{2(n+b), 1-\alpha}^{2}$ function which represents the lower $1-\alpha$ percentile of $\chi_{2(n+b)}^{2}$, then

$$
P\left(\frac{t}{\theta^{2}} \leq \chi_{2(n+b), 1-\alpha}^{2} \mid \underset{\sim}{x}\right)=1-\alpha,
$$

where $\underset{\sim}{x}=\left(x_{U(1)}, x_{U(2)}, \ldots, x_{U(n)}\right)$,

$$
\begin{aligned}
\Longrightarrow P\left(C_{L} \geq \sqrt{\frac{\pi}{4-\pi}}-\left(\sqrt{\frac{\pi}{4-\pi}}-\widehat{C}_{L, \mathrm{BS}}\right)\right. \\
\left.\times \frac{\Gamma(n+b-1 / 2)}{\Gamma(n+b)}\left(\frac{\chi_{2(n+b), 1-\alpha}^{2}}{2}\right)^{1 / 2} \underset{\sim}{\underset{x}{x}}\right)
\end{aligned}
$$

$=1-\alpha$,

where $C_{L}$ as the definition of (5).

From (23), we obtain that a $100(1-\alpha) \%$ one-sided credible interval for $C_{L}$ is given by

$$
\begin{aligned}
C_{L} \geq & \sqrt{\frac{\pi}{4-\pi}}-\left(\sqrt{\frac{\pi}{4-\pi}}-\widehat{C}_{L, \mathrm{BS}}\right) \\
& \times \frac{\Gamma(n+b-1 / 2)}{\Gamma(n+b)}\left(\frac{\chi_{2(n+b), 1-\alpha}^{2}}{2}\right)^{1 / 2},
\end{aligned}
$$

where $\widehat{C}_{L, B S}$ is given by (22).

Therefore, the $100(1-\alpha) \%$ lower credible interval bound for $C_{L}$ can be written as

$$
\begin{aligned}
\underline{L B}_{\mathrm{BS}}= & \sqrt{\frac{\pi}{4-\pi}}-\left(\sqrt{\frac{\pi}{4-\pi}}-\widehat{C}_{L, \mathrm{BS}}\right) \\
& \times \frac{\Gamma(n+b-1 / 2)}{\Gamma(n+b)}\left(\frac{\chi_{2(n+b), 1-\alpha}^{2}}{2}\right)^{1 / 2},
\end{aligned}
$$


where $\widehat{C}_{L, B S}$ is given by (22), and $b$ is a parameter of prior distribution with density as (15).

The managers can use the one-sided credible interval to determine whether the product performance adheres to the required level. The proposed testing procedure of $C_{L}$ with $\widehat{C}_{L, \mathrm{BS}}$ can be organized as follows.

Step 1. Determine the lower lifetime limit $L$ for products and the performance index value $c_{0}$, then the testing null hypothesis $H_{0}: C_{L} \leq c_{0}$ and the alternative hypothesis $H_{1}: C_{L}>c_{0}$ are constructed.

Step 2. Specify a significance level $\alpha$.

Step 3. Given the parameters $a$ and $b$ of prior distribution, the upper record values $\underset{\sim}{x}=\left(x_{U(1)}, x_{U(2)}, \ldots, x_{U(n)}\right)$, the lower lifetime limit $L$, and the significance level $\alpha$, then we can calculate the $100(1-\alpha) \%$ one-sided credible interval $\left[\underline{\mathrm{LB}}_{\mathrm{BS}}, \infty\right)$ for $C_{L}$, where $\underline{\mathrm{LB}}_{\mathrm{BS}}$ as the definition of (25).

Step 4. The decision rule of statistical test is provided as follows:

(1) if the performance index value $c_{0} \notin\left[\underline{L B}_{B S}, \infty\right)$, then we will reject $H_{0}$. It is concluded that the lifetime performance index of products meets the required level;

(2) if the performance index value $c_{0} \in\left[\underline{\mathrm{LB}}_{\mathrm{BS}}, \infty\right)$, then we do not reject $H_{0}$. It is concluded that the lifetime performance index of products does not meet the required level.

5.2. Testing Procedure for the Lifetime Performance Index $C_{L}$ with the Bayesian Estimator under Linear Exponential Loss Function. Let $X$ be the lifetime of such a product, and $X$ has a Rayleigh distribution with the p.d.f. as given by (2). We consider the linear exponential (LINEX) loss function as (also see [18-23])

$$
L(\Delta) \propto e^{c \Delta}-c \Delta-1,
$$

where $\Delta=\left(\theta^{*}-\theta\right), c \neq 0$.

In this paper, we suppose that

$$
\Delta_{1}=\left(\frac{\theta^{*}}{\theta}\right)^{2}-1,
$$

where $\theta^{*}$ is an estimator of $\theta$.

By using (26)-(27), the posterior expectation of LINEX loss function $L\left(\Delta_{1}\right)$ is

$$
\begin{aligned}
E\left[L\left(\Delta_{1}\right) \mid \underset{\sim}{x}\right]= & e^{-c} E\left[e^{c\left(\theta^{*} / \theta\right)_{2}} \mid \underset{\sim}{x}\right] \\
& -c E\left[\left(\frac{\theta^{*}}{\theta}\right)^{2}-1 \mid \underset{\sim}{x}\right]-1,
\end{aligned}
$$

where $\underset{\sim}{x}=\left(x_{U(1)}, x_{U(2)}, \ldots, x_{U(n)}\right)$.
The value of $\theta^{*}$ that minimizes $E\left[L\left(\Delta_{1}\right) \mid \underset{\sim}{x}\right]$ denoted by $\widehat{\theta}_{\mathrm{BL}}$ is obtained by solving the equation:

$$
\begin{aligned}
\frac{d E\left[L\left(\Delta_{1}\right) \mid \underset{\sim}{x}\right]}{d \theta^{*}}= & e^{-c} E\left[e^{c\left(\theta^{*} / \theta\right)_{2}} \cdot \frac{2 c \theta^{*}}{\theta^{2}} \mid \underset{\sim}{x}\right] \\
& -c E\left(\frac{2 \theta^{*}}{\theta^{2}} \mid \underset{\sim}{x}\right)=0 ;
\end{aligned}
$$

that is, $\widehat{\theta}_{\mathrm{BL}}$ is the solution to the following equation:

$$
E\left[\frac{\widehat{\theta}_{\mathrm{BL}}}{\theta^{2}} e^{c\left(\widehat{\theta}_{\mathrm{BL}} / \theta\right)_{2}} \mid \underset{\sim}{x}\right]=e^{c} E\left(\frac{\widehat{\theta}_{\mathrm{BL}}}{\theta^{2}} \mid \underset{\sim}{x}\right) .
$$

By using (18) and (30), we have

$$
\begin{array}{r}
2 \widehat{\theta}_{\mathrm{BL}}(n+b) \cdot \frac{t^{n+b}}{\left(t-2 c \widehat{\theta}_{\mathrm{BL}}^{2}\right)^{n+b+1}}=e^{c} \widehat{\theta}_{\mathrm{BL}} \frac{2(n+b)}{t}, \\
\text { where } t=x_{U(n)}^{2}+a .
\end{array}
$$

Hence, the Bayesian estimator of $\theta$ under the LINEX loss function is given by

$$
\widehat{\theta}_{\mathrm{BL}}=\left[\frac{T}{2 c}\left(1-e^{-c /(n+b+1)}\right)\right]^{1 / 2},
$$

where $T=X_{U(n)}^{2}+a, a$, and $b$ are the parameters of prior distribution with density as (15).

By using (5) and the Bayesian estimator $\widehat{\theta}_{\mathrm{BL}}$ as $(32), \widehat{C}_{L, \mathrm{BL}}$ based on the Bayesian estimator $\widehat{\theta}_{\mathrm{BL}}$ of $\theta$ is given by

$$
\begin{aligned}
\widehat{C}_{L, \mathrm{BL}} & =\sqrt{\frac{\pi}{4-\pi}}-\sqrt{\frac{2}{4-\pi}} \frac{L}{\widehat{\theta}_{\mathrm{BL}}} \\
& =\sqrt{\frac{\pi}{4-\pi}}-\sqrt{\frac{2}{4-\pi}} L\left[\frac{T}{2 c}\left(1-e^{-c /(n+b+1)}\right)\right]^{-1 / 2},
\end{aligned}
$$

where $T=X_{U(n)}^{2}+a, a$, and $b$ are the parameters of prior distribution with density as (15).

We construct a statistical testing procedure to assess whether the lifetime performance index adheres to the required level. The one-sided credible confidence interval for $C_{L}$ is obtained using the pivotal quantity $t /\left.\theta^{2}\right|_{\sim=\left(x_{U(1)}, \ldots, x_{U(n)}\right)}$. By using the pivotal quantity $t /\left.\theta^{2}\right|_{x=\left(x_{U(1)}, \ldots, x_{U(n)}\right)}$, given the specified significance level $\alpha$, the level $100(1-\alpha) \%$ one-sided credible interval for $C_{L}$ can be derived as follows. 


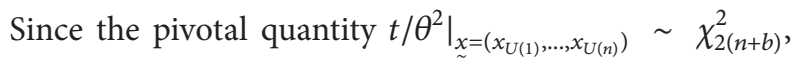
and $\chi_{2(n+b), 1-\alpha}^{2}$ function which represents the lower $1-\alpha$ percentile of $\chi_{2(n+b)}^{2}$, then

$$
P\left(\frac{t}{\theta^{2}} \leq \chi_{2(n+b), 1-\alpha}^{2} \mid \underset{\sim}{x}\right)=1-\alpha
$$

where $\underset{\sim}{x}=\left(x_{U(1)}, x_{U(2)}, \ldots, x_{U(n)}\right)$,

$$
\begin{aligned}
\Longrightarrow P( & C_{L} \geq \sqrt{\frac{\pi}{4-\pi}}-\left(\sqrt{\frac{\pi}{4-\pi}}-\widehat{C}_{L, \mathrm{BL}}\right) \\
\times & {\left.\left[\frac{1}{2 c}\left(1-e^{-c /(n+b+1)}\right) \chi_{2(n+b), 1-\alpha}^{2}\right]^{1 / 2} \underset{\sim}{\mid \underset{\sim}{x}}\right) }
\end{aligned}
$$

$=1-\alpha$,

where $C_{L}$ as the definition of (5).

From (34), we obtain that a $100(1-\alpha) \%$ one-sided credible interval for $C_{L}$ is

$$
\begin{aligned}
C_{L} \geq & \sqrt{\frac{\pi}{4-\pi}}-\left(\sqrt{\frac{\pi}{4-\pi}}-\widehat{C}_{L, \mathrm{BL}}\right) \\
& \times\left[\frac{1}{2 c}\left(1-e^{-c /(n+b+1)}\right) \chi_{2(n+b), 1-\alpha}^{2}\right]^{1 / 2},
\end{aligned}
$$

where $\widehat{C}_{L, \mathrm{BL}}$ is given by (33).

Therefore, the $100(1-\alpha) \%$ lower credible interval bound for $C_{L}$ can be written as

$$
\begin{aligned}
\underline{\mathrm{LB}}_{\mathrm{BL}}= & \sqrt{\frac{\pi}{4-\pi}}-\left(\sqrt{\frac{\pi}{4-\pi}}-\widehat{C}_{L, \mathrm{BL}}\right) \\
& \times\left[\frac{1}{2 c}\left(1-e^{-c /(n+b+1)}\right) \chi_{2(n+b), 1-\alpha}^{2}\right]^{1 / 2},
\end{aligned}
$$

where $\widehat{C}_{L, B L}$ is given by (33), and $b$ is a parameter of prior distribution with density as (15).

The managers can use the one-sided credible interval to determine whether the product performance attains to the required level. The proposed testing procedure of $C_{L}$ with $\widehat{C}_{L, B L}$ can be organized as follows.

Step 1. Determine the lower lifetime limit $L$ for products and the performance index value $c_{0}$, then the testing null hypothesis $H_{0}: C_{L} \leq c_{0}$ and the alternative hypothesis $H_{1}: C_{L}>c_{0}$ are constructed.

Step 2. Specify a significance level $\alpha$.

Step 3. Given the parameters $a$ and $b$ of prior distribution, the parameter $c$ of LINEX loss function, the upper record values $\underset{\sim}{x}=\left(x_{U(1)}, x_{U(2)}, \ldots, x_{U(n)}\right)$, the lower lifetime limit $L$, and the significance level $\alpha$, then we can calculate the $100(1-\alpha) \%$ one-sided credible interval $\left[\underline{\mathrm{LB}}_{\mathrm{BL}}, \infty\right)$ for $C_{L}$, where $\underline{\mathrm{LB}}_{\mathrm{BL}}$ as the definition of (36).

Step 4. The decision rule of statistical test is provided as follows:
(1) if the performance index value $c_{0} \notin\left[\underline{L B}_{B L}, \infty\right)$, then we will reject $H_{0}$. It is concluded that the lifetime performance index of products meets the required level;

(2) if the performance index value $c_{0} \in\left[\underline{L B}_{B L}, \infty\right)$, then we do not reject $H_{0}$. It is concluded that the lifetime performance index of products does not meet the required level.

5.3. Testing Procedure for the Lifetime Performance Index $C_{L}$ with the Bayesian Estimator under General Entropy Loss Function. Let $X$ be the lifetime of such a product, and $X$ has a Rayleigh distribution with the p.d.f. as given by (2). We consider the general entropy (GE) loss function (also see $[12,20])$ :

$$
L\left(\theta^{*}, \theta\right) \propto\left(\frac{\theta^{*}}{\theta}\right)^{q}-q \log \left(\frac{\theta^{*}}{\theta}\right)-1,
$$

whose minimum occurs at $\theta^{*}=\theta$.

The loss function is a generalization of entropy loss used by several authors (e.g., Dyer and Liu [24], Soliman [25], and Soliman and Elkahlout [26]) where the shape parameter $q=$ 1 . When $q>0$, a positive error $\left(\theta^{*}>\theta\right)$ causes more serious consequences than a negative error. The Bayesian estimator $\widehat{\theta}_{\mathrm{BG}}$ of $\theta$ under GE loss function is given by

$$
\widehat{\theta}_{\mathrm{BG}}=\left[E\left(\theta^{-q} \mid x_{U(1)}, \ldots, x_{U(n)}\right)\right]^{-1 / q} \text {. }
$$

By using (18) and (38), then the Bayesian estimator $\widehat{\theta}_{\mathrm{BG}}$ of $\theta$ is derived as follows:

$$
\begin{aligned}
E\left(\theta^{-q}\right. & \left.\mid x_{U(1)}, \ldots, x_{U(n)}\right) \\
& =\int_{0}^{\infty} \theta^{-q} p_{1}\left(\theta \mid x_{U(1)}, \ldots, x_{U(n)}\right) d \theta \\
& =\left(\frac{t}{2}\right)^{-q / 2} \cdot \frac{\Gamma(n+b+q / 2)}{\Gamma(n+b)},
\end{aligned}
$$

where $t=x_{U(n)}^{2}+a$. Hence, the Bayesian estimator $\widehat{\theta}_{\mathrm{BG}}$ of $\theta$ under the GE loss function is given by

$$
\widehat{\theta}_{\mathrm{BG}}=\left(\frac{T}{2}\right)^{1 / 2}\left[\frac{\Gamma(n+b+q / 2)}{\Gamma(n+b)}\right]^{-1 / q},
$$

where $T=X_{U(n)}^{2}+a, a$, and $b$ are the parameters of prior distribution with density as (15).

By using (5) and the Bayesian estimator $\widehat{\theta}_{\mathrm{BG}}$ as $(40), \widehat{C}_{L, \mathrm{BG}}$ based on the Bayesian estimator $\widehat{\theta}_{\mathrm{BG}}$ of $\theta$ is given by

$$
\begin{aligned}
\widehat{C}_{L, B G}= & \sqrt{\frac{\pi}{4-\pi}}-\sqrt{\frac{2}{4-\pi}} \frac{L}{\widehat{\theta}_{\mathrm{BG}}} \\
= & \sqrt{\frac{\pi}{4-\pi}}-\sqrt{\frac{2}{4-\pi}} \cdot L \\
& \cdot\left[\left(\frac{T}{2}\right)^{-q / 2} \frac{\Gamma(n+b+q / 2)}{\Gamma(n+b)}\right]^{1 / q},
\end{aligned}
$$


where $T=X_{U(n)}^{2}+a, a$, and $b$ are the parameters of prior distribution with density as (15).

We construct a statistical testing procedure to assess whether the lifetime performance index adheres to the required level. The one-sided credible confidence interval for

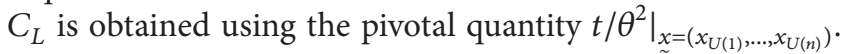

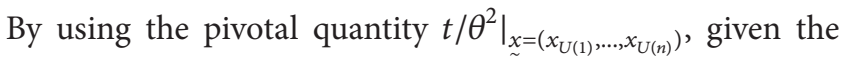
specified significance level $\alpha$, the level $100(1-\alpha) \%$ one-sided credible interval for $C_{L}$ can be derived as follows.

Since the pivotal quantity $t /\left.\theta^{2}\right|_{\sim=\left(x_{U(1)}, \ldots, x_{U(n)}\right)} \sim \chi_{2(n+b)}^{2}$, and $\chi_{2(n+b), 1-\alpha}^{2}$ function which represents the lower $1-\alpha$ percentile of $\chi_{2(n+b)}^{2}$, then

$$
P\left(\frac{t}{\theta^{2}} \leq \chi_{2(n+b), 1-\alpha}^{2} \mid \underset{\sim}{x}\right)=1-\alpha,
$$

where $\underset{\sim}{x}=\left(x_{U(1)}, x_{U(2)}, \ldots, x_{U(n)}\right)$,

$$
\begin{aligned}
\Longrightarrow P\left(C_{L} \geq \sqrt{\frac{\pi}{4-\pi}}-\left(\sqrt{\frac{\pi}{4-\pi}}-\widehat{C}_{L, \mathrm{BG}}\right)\right. \\
\left.\times\left[\frac{\Gamma(n+b+q / 2)}{\Gamma(n+b)}\right]^{-1 / q}\left(\frac{\chi_{2(n+b), 1-\alpha}^{2}}{2}\right)^{1 / 2} \mid \underset{\sim}{x}\right)
\end{aligned}
$$

$=1-\alpha$

where $C_{L}$ as the definition of (5).

From (42), we obtain that a $100(1-\alpha) \%$ one-sided credible interval for $C_{L}$ is

$$
\begin{aligned}
C_{L} \geq & \sqrt{\frac{\pi}{4-\pi}}-\left(\sqrt{\frac{\pi}{4-\pi}}-\widehat{C}_{L, \mathrm{BG}}\right) \\
& \times\left[\frac{\Gamma(n+b+q / 2)}{\Gamma(n+b)}\right]^{-1 / q}\left(\frac{\chi_{2(n+b), 1-\alpha}^{2}}{2}\right)^{1 / 2},
\end{aligned}
$$

where $\widehat{C}_{L, B G}$ is given by (41).

Therefore, the $100(1-\alpha) \%$ lower credible interval bound for $C_{L}$ can be written as

$$
\begin{aligned}
\underline{\mathrm{LB}}_{\mathrm{BG}}= & \sqrt{\frac{\pi}{4-\pi}}-\left(\sqrt{\frac{\pi}{4-\pi}}-\widehat{C}_{L, \mathrm{BG}}\right) \\
& \times\left[\frac{\Gamma(n+b+q / 2)}{\Gamma(n+b)}\right]^{-1 / q}\left(\frac{\chi_{2(n+b), 1-\alpha}^{2}}{2}\right)^{1 / 2},
\end{aligned}
$$

where $\widehat{C}_{L, \mathrm{BG}}$ is given by (41), $\alpha$ is the specified significance level, and $b$ is a parameter of prior distribution with density as (15).

The managers can use the one-sided credible interval to determine whether the product performance attains to the required level. The proposed testing procedure of $C_{L}$ with $\widehat{C}_{L, B G}$ can be organized as follows.

Step 1. Determine the lower lifetime limit $L$ for products and the performance index value $c_{0}$, then the testing null hypothesis $H_{0}: C_{L} \leq c_{0}$ and the alternative hypothesis $H_{1}: C_{L}>c_{0}$ are constructed.

Step 2. Specify a significance level $\alpha$.

Step 3. Given the parameters $a$ and $b$ of prior distribution, the parameter $q$ of GE loss function, the upper record values $\underset{\sim}{x}=\left(x_{U(1)}, x_{U(2)}, \ldots, x_{U(n)}\right)$, the lower lifetime limit $L$, and the significance level $\alpha$, then we can calculate the $100(1-\alpha) \%$ one-sided credible interval $\left[\underline{\mathrm{LB}}_{\mathrm{BG}}, \infty\right)$ for $C_{L}$, where $\underline{\mathrm{LB}}_{\mathrm{BG}}$ as the definition of (44).

Step 4. The decision rule of statistical test is provided as follows:

(1) if the performance index value $c_{0} \notin\left[\underline{\mathrm{LB}}_{\mathrm{BG}}, \infty\right)$, then we will reject $H_{0}$. It is concluded that the lifetime performance index of products meets the required level;

(2) if the performance index value $c_{0} \in\left[\underline{\mathrm{LB}}_{\mathrm{BG}}, \infty\right)$, then we do not reject $H_{0}$. It is concluded that the lifetime performance index of products does not meet the required level.

\section{The Monte Carlo Simulation Algorithm of Confidence (or Credible) Level}

In this section, we will report the results of a simulation study for confidence (or credible) level $(1-\alpha)$ based on a $100(1-\alpha) \%$ one-sided confidence (or credible) interval of the lifetime performance index $C_{L}$. We considered $\alpha=0.05$ and then generated samples from the Rayleigh distribution with p.d.f. as in (2) with respect to the record values.

(i) The Monte Carlo simulation algorithm of confidence level $(1-\alpha)$ for $C_{L}$ under MLE is given in the following steps.

Step 1. Given $n, a, b, L$, and $\alpha$, where $a>0, b>0$.

Step 2. For the values of prior parameters $(a, b)$, use (15) to generate $\theta$ from the square-root inverted-gamma distribution.

Step 3.

(a) The generation of data $\left(Z_{1}, Z_{2}, \ldots, Z_{n}\right)$ is by standard exponential distribution with parameter 1 .

(b) Set $Y_{1}^{2}=2 \theta^{2} Z_{1}$ and $Y_{i}^{2}=2 \theta^{2} Z_{i}+Y_{i-1}^{2}$, for $i=2, \ldots, n$.

$Y_{1}=\sqrt{2 \theta^{2} Z_{1}}, \quad Y_{i}=\sqrt{2 \theta^{2} Z_{i}+Y_{i-1}^{2}}, \quad$ for $i=2, \ldots, n$.

$\left(Y_{1}, Y_{2}, \ldots, Y_{n}\right)$ are the upper record values from the Rayleigh distribution.

(c) The value of $\underline{\mathrm{LB}}_{\mathrm{MLE}}$ is calculated by

$\underline{\mathrm{LB}}_{\mathrm{MLE}}=\sqrt{\frac{\pi}{4-\pi}}-\left(\sqrt{\frac{\pi}{4-\pi}}-\widehat{C}_{L, \mathrm{MLE}}\right)\left(\frac{\chi_{2 n, 1-\alpha}^{2}}{2 n}\right)^{1 / 2}$, 
where $\widehat{C}_{L, M L E}$ is given by (11) and $\chi_{2 n, 1-\alpha}^{2}$ function which represents the lower $1-\alpha$ percentile of $\chi_{2 n}^{2}$.

(d) If $C_{L} \geq \underline{\mathrm{LB}}_{\mathrm{MLE}}$, then count $=1$; else count $=0$.

Step 4.

(a) Step 3 is repeated 1000 times.

(b) The estimation of confidence level $(1-\alpha)$ is $(\widehat{1-\alpha})=$ (total count)/1000.

Step 5.

(a) Repeat Step 2-Step 4 for 100 times, then we can get the 100 estimations of confidence level as follows:

$$
(\widehat{1-\alpha})_{1},(\widehat{1-\alpha})_{2}, \ldots,(\widehat{1-\alpha})_{100} .
$$

(b) The average empirical confidence level $\overline{1-\alpha}$ of $(\widehat{1-\alpha})_{i}, i=1, \ldots, 100$; that is, $\overline{1-\alpha}=$ $(1 / 100) \sum_{i=1}^{100}(\widehat{1-\alpha})_{i}$.

(c) The sample mean square error (SMSE) of $(\widehat{1-\alpha})_{1},(\overline{1-\alpha})_{2}, \ldots,(\widehat{1-\alpha})_{100}$ SMSE $=(1 / 100)$ $\times \sum_{i=1}^{100}\left[(\widehat{1-\alpha})_{i}-(1-\alpha)\right]^{2}$.

The results of simulation are summarized in Table 2 based on $L=1.0$, the different value of sample size $n$, prior parameter $(a, b)=(2,5),(6,1.5)$, and $(2,2)$ at $\alpha=0.05$, respectively. The scope of SMSE is between 0.00413 and 0.00593 .

Step 1. Given $n, a, b, L$, and $\alpha$, where $a>0, b>0$.

Step 2. For the values of prior parameters $(a, b)$, use (15) to generate $\theta$ from the square-root inverted-gamma distribution.

Step 3.

(a) The generation of data $\left(Z_{1}, Z_{2}, \ldots, Z_{n}\right)$ is by standard exponential distribution with parameter 1 .

(b) Set $Y_{1}^{2}=2 \theta^{2} Z_{1}$ and $Y_{i}^{2}=2 \theta^{2} Z_{i}+Y_{i-1}^{2}$, for $i=2, \ldots, n$.

$Y_{1}=\sqrt{2 \theta^{2} Z_{1}}, \quad Y_{i}=\sqrt{2 \theta^{2} Z_{i}+Y_{i-1}^{2}}, \quad$ for $i=2, \ldots, n$.

$\left(Y_{1}, Y_{2}, \ldots, Y_{n}\right)$ are the upper record values from the Rayleigh distribution.

(c) The values of $\underline{\mathrm{LB}}_{\mathrm{BS}}, \underline{\mathrm{LB}}_{\mathrm{BL}}$, and $\underline{\mathrm{LB}}_{\mathrm{BG}}$ are calculated by (25), (36), and (44), respectively.

(d) If $C_{L} \geq \underline{L B}$, then count $=1$; else count $=0$, where $\underline{\mathrm{LB}}=$ $\underline{\mathrm{LB}}_{\mathrm{BS}}$ or $\underline{\mathrm{LB}}_{\mathrm{BL}}$ or $\underline{\mathrm{LB}}_{\mathrm{BG}}$

Step 4.

(a) Step 3 is repeated 1000 times.

(b) The estimation of credible level $(1-\alpha)$ is $(\widehat{1-\alpha})=$ (total count)/1000.
Step 5.

(a) Repeat Step 2-Step 4 for 100 times, then we can get the 100 estimations of credible level as follows:

$$
(\widehat{1-\alpha})_{1},(\widehat{1-\alpha})_{2}, \ldots,(\widehat{1-\alpha})_{100}
$$

(b) The average empirical credible level $\overline{1-\alpha}$ of $(\widehat{1-\alpha})_{i}$, $i=1, \ldots, 100$; that is, $\overline{1-\alpha}=(1 / 100) \sum_{i=1}^{100}(\widehat{1-\alpha})_{i}$.

(c) The sample mean square error (SMSE) of $(\widehat{1-\alpha})_{1},(\overline{1-\alpha})_{2}, \ldots,(\widehat{1-\alpha})_{100}$ SMSE $=(1 / 100)$ $\times \sum_{i=1}^{100}\left[(\overline{1-\alpha})_{i}-(1-\alpha)\right]^{2}$.

Based on $L=1.0$, the different value of sample size $n$, prior parameter $(a, b)=\{(2,5),(6,1.5),(2,2)\}$, and $\alpha=$ 0.05 , the results of simulation are summarized in Tables 3-5 under SE loss function, LINEX loss function with parameter $c=\{-0.5,0.5,1.5\}$, and GE loss function with parameter $q=\{3,5,8\}$, respectively. The following points can be drawn.

(a) All of the SMSEs are small enough and the scope of SMSE is between 0.00346 and 0.00467 .

(b) Fix the size $n$, comparison of prior parameter $(a, b)=$ $(2,5),(6,1.5)$, and $(2,2)$ as follows:

(i) the values of SMSE with prior parameter $(a, b)=$ $(6,1.5)$ and $(2,5)$ are smaller than $(a, b)=(2,2)$, respectively;

(ii) a comparison of prior parameter $(a, b)=(2,5)$, and $(2,2)$; that is, fix prior parameter $a$, if prior parameter $b$ increases, then it can be seen that the SMSE will decrease.

(c) In Table 4, fix the size $n$ and the prior parameter $(a, b)$, comparison the parameter of LINEX loss function $c=$ $\{-0.5,0.5,1.5\}$ as follows:

the values of SMSE with prior parameter $c=0.5,1.5$, and -1.5 are the same as the values of SMSE in Table 3.

(d) In Table 5, fix the prior parameter $(a, b)$, comparison of GE loss function parameter $q=\{3,5,8\}$ as follows: the values of SMSE with prior parameter $q=3,5$, and 8 are the same as the values of SMSE in Table 3.

Hence, these results from simulation studies illustrate that the performance of our proposed method is acceptable. Moreover, we suggest that the prior parameter $(a, b)=(6,1.5)$ and $(2,5)$ is appropriate for the square-root inverted-gamma distribution.

\section{Numerical Example}

In this section, we propose the new hypothesis testing procedures to a real-life data. In the following example, we discuss a real-life data for 25 ball bearings to illustrate the use of the new hypothesis testing procedures in the lifetime performance of ball bearings. The proposed testing procedures not only can handle nonnormal lifetime data, but also can handle the upper record values. 
TABLE 2: Average empirical confidence level $(1-\alpha)$ for $C_{L}$ under MLE when $\alpha=0.05$.

\begin{tabular}{lccr}
\hline$n$ & $a=2, b=5$ & $a=6, b=1.5$ & $a=2, b=2$ \\
\hline 5 & $0.93653(0.00462)$ & $0.93482(0.00467)$ & $0.93577(0.00507)$ \\
10 & $0.93643(0.00449)$ & $0.93581(0.00413)$ & $0.93450(0.00593)$ \\
15 & $0.93523(0.00489)$ & $0.93557(0.00503)$ & $0.93463(0.00516)$ \\
\hline
\end{tabular}

$n$ denotes the sample size; the values in parentheses are sample mean square error of $(\widehat{1-\alpha})$.

TABLE 3: Average empirical credible level $(1-\alpha)$ for $C_{L}$ under SE loss function when $\alpha=0.05$.

\begin{tabular}{lccc}
\hline$n$ & $a=2, b=5$ & $a=6, b=1.5$ & $a=2, b=2$ \\
\hline 5 & $0.94357(0.00386)$ & $0.93760(0.00386)$ & $0.93925(0.00395)$ \\
10 & $0.94244(0.00359)$ & $0.93704(0.00359)$ & $0.93664(0.00462)$ \\
15 & $0.94096(0.00346)$ & $0.93619(0.00426)$ & $0.93635(0.00467)$ \\
\hline
\end{tabular}

$n$ denotes the sample size; the values in parentheses are sample mean square error of $(\widehat{1-\alpha})$.

TABLE 4: Average empirical credible level $(1-\alpha)$ for $C_{L}$ under LINEX loss function when $\alpha=0.05$.

\begin{tabular}{ccccc}
\hline$n$ & $c$ & $a=2, b=5$ & $a=6, b=1.5$ & $a=2, b=2$ \\
\hline \multirow{2}{*}{5} & -0.5 & $0.94357(0.00386)$ & $0.93760(0.00386)$ & $0.93925(0.00395)$ \\
& 0.5 & $0.94357(0.00386)$ & $0.93760(0.00386)$ & $0.93925(0.00395)$ \\
& 1.5 & $0.94357(0.00386)$ & $0.93760(0.00386)$ & $0.93925(0.00395)$ \\
\hline \multirow{3}{*}{10} & -0.5 & $0.94244(0.00359)$ & $0.93704(0.00359)$ & $0.93664(0.00462)$ \\
& 0.5 & $0.94244(0.00359)$ & $0.93704(0.00359)$ & $0.93664(0.00462)$ \\
& 1.5 & $0.94244(0.00359)$ & $0.93704(0.00359)$ & $0.93664(0.00462)$ \\
\hline \multirow{3}{*}{15} & -0.5 & $0.94096(0.00346)$ & $0.93619(0.00426)$ & $0.93635(0.00467)$ \\
& 0.5 & $0.94096(0.00346)$ & $0.93619(0.00426)$ & $0.93635(0.00467)$ \\
& 1.5 & $0.94096(0.00346)$ & $0.93619(0.00426)$ & $0.93635(0.00467)$ \\
\hline
\end{tabular}

$n$ denotes the sample size; the values in parentheses are sample mean square error of $(\widehat{1-\alpha})$.

TABLE 5: Average empirical credible level $(1-\alpha)$ for $C_{L}$ under GE loss function when $\alpha=0.05$.

\begin{tabular}{|c|c|c|c|c|}
\hline$n$ & $q$ & $a=2, b=5$ & $a=6, b=1.5$ & $a=2, b=2$ \\
\hline & 3 & $0.94357(0.00386)$ & $0.93760(0.00386)$ & $0.93925(0.00395)$ \\
\hline \multirow[t]{3}{*}{5} & 5 & $0.94357(0.00386)$ & $0.93760(0.00386)$ & $0.93925(0.00395)$ \\
\hline & 8 & $0.94357(0.00386)$ & $0.93760(0.00386)$ & $0.93925(0.00395)$ \\
\hline & 3 & $0.94244(0.00359)$ & $0.93704(0.00359)$ & $0.93664(0.00462)$ \\
\hline \multirow[t]{3}{*}{10} & 5 & $0.94244(0.00359)$ & $0.93704(0.00359)$ & $0.93664(0.00462)$ \\
\hline & 8 & $0.94244(0.00359)$ & $0.93704(0.00359)$ & $0.93664(0.00462)$ \\
\hline & 3 & $0.94096(0.00346)$ & $0.93619(0.00426)$ & $0.93635(0.00467)$ \\
\hline \multirow[t]{2}{*}{15} & 5 & $0.94096(0.00346)$ & $0.93619(0.00426)$ & $0.93635(0.00467)$ \\
\hline & 8 & $0.94096(0.00346)$ & $0.93619(0.00426)$ & $0.93635(0.00467)$ \\
\hline
\end{tabular}

$n$ denotes the sample size; the values in parentheses are sample mean square error of $(\widehat{1-\alpha})$.

Example. The data is the failure times of 25 ball bearings in endurance test. The 25 observations are the number of million revolutions before failure for each of the ball bearings. The data come from Caroni [27] as follows:

$67.80,67.80,67.80,68.64,33.00,68.64,98.64,128.04,42.12$, 28.92, 45.60, 51.84, 55.56, 173.40, 48.48, 17.88, 93.12, 54.12, $41.52,51.96,127.92,84.12,105.12,105.84$, and 68.88 .

Raqab and Madi [28] and Lee [29] indicated that a oneparameter Rayleigh distribution is acceptable for these data. In addition, we also have a way to test the hypothesis that the failure data come from the Rayleigh distribution. The testing hypothesis $H_{0}: X$ comes from a Rayleigh distribution versus $H_{1}: X$ does not come from a Rayleigh distribution is constructed under significance level $\alpha=0.05$.

From a probability plot of Figure 1 by using the Minitab Statistical Software, we can conclude the operational lifetimes data of 25 ball bearings from the Rayleigh distribution which is the Weibull distribution with the shape 2 (also see Lee et al. [14]). For informative prior, we use the prior information: $E(\theta)=\hat{\theta}_{\mathrm{MLE}} \doteq 54.834$ and $\operatorname{Var}(\theta)=0.2$, giving the prior parameter values as $(a=6.014, b=1.001)$.

Here, if only the upper record values have been observed, these are $\left\{x_{U(i)}, i=1, \ldots, 5(=n)\right\}=\{67.80,68.64,98.64$, $128.04,173.40\}$. 


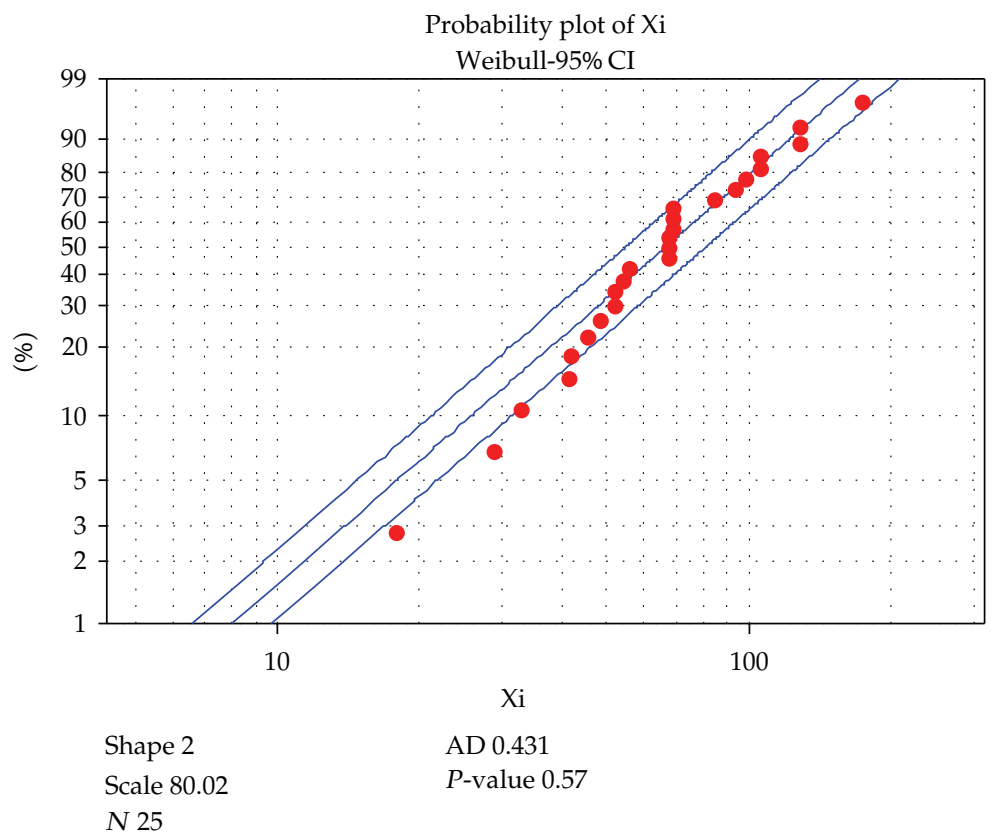

FIgURE 1: Probability plot for failure times of 25 ball bearings data.

(1) The proposed testing procedure of $C_{L}$ with $\widehat{C}_{L, M L E}$ is stated as follows.

Step 1. The record values from the above data are $\left(x_{U(i)}, i=\right.$ $1,2,3,4,5)=(67.80,68.64,98.64,128.04,173.40)$.

Step 2. The lower specification limit $L$ is assumed to be 23.37. The deal with the product managers' concerns regarding lifetime performance and the conforming rate $P_{r}$ of products is required to exceed 80 percent. Referring to Table $1, C_{L}$ is required to exceed 0.90 . Thus, the performance index value is set at $c_{0}=0.90$. The testing hypothesis $H_{0}: C_{L} \leq 0.90$ versus $H_{1}: C_{L}>0.90$ is constructed.

Step 3. Specify a significance level $\alpha=0.05$.

Step 4. We can calculate that the $95 \%$ lower confidence interval bound for $C_{L}$, where

$$
\begin{aligned}
\underline{\mathrm{LB}}_{\mathrm{MLE}} & =\sqrt{\frac{\pi}{4-\pi}}-\left(\sqrt{\frac{\pi}{4-\pi}}-\widehat{C}_{L, \mathrm{MLE}}\right)\left(\frac{\chi_{2 n, 1-\alpha}^{2}}{2 n}\right)^{1 / 2} \\
& =\sqrt{\frac{\pi}{4-\pi}}-\left(\sqrt{\frac{\pi}{4-\pi}}-1.26251254\right)\left(\frac{\chi_{10,0.95}^{2}}{10}\right)^{1 / 2} \\
& =1.03285 .
\end{aligned}
$$

So, the $95 \%$ one-sided confidence interval for $C_{L}$ is $\left[\underline{\mathrm{LB}}_{\mathrm{MLE}}, \infty\right)=[1.03285, \infty)$.

Step 5. Because of the performance index value $c_{0}=0.90 \notin$ $\left[\underline{\mathrm{LB}}_{\mathrm{MLE}}, \infty\right)$, we reject the null hypothesis $H_{0}: C_{L} \leq 0.90$.

Thus, we can conclude that the lifetime performance index of data meets the required level.
(2) The proposed testing procedures of $C_{L}$ with $\widehat{C}_{L, B S}$, $\widehat{C}_{L, \mathrm{BL}}$, and $\widehat{C}_{L, \mathrm{BG}}$ are stated as follows.

Step 1. The record values from the above data are $\left(x_{U(i)}, i=\right.$ $1,2,3,4,5)=(67.80,68.64,98.64,128.04,173.40)$.

Step 2. The lower specification limit $L$ is assumed to be 23.37 . The deal with the product managers' concerns regarding lifetime performance and the conforming rate $P_{r}$ of products is required to exceed 80 percent. Referring to Table $1, C_{L}$ is required to exceed 0.90 . Thus, the performance index value is set at $c_{0}=0.90$. The testing hypothesis $H_{0}: C_{L} \leq 0.90$ versus $H_{1}: C_{L}>0.90$ is constructed.

Step 3. Specify a significance level $\alpha=0.05$, the parameter of LINEX loss function $c=0.5$, and the parameter of GE loss function $q=2$.

Step 4. We can calculate the $95 \%$ lower credible interval bound for $C_{L}$, where

$$
\begin{aligned}
\underline{L B}_{\mathrm{BS}}= & \sqrt{\frac{\pi}{4-\pi}}-\left(\sqrt{\frac{\pi}{4-\pi}}-\widehat{C}_{L, \mathrm{BS}}\right) \\
& \times \frac{\Gamma(n+b-1 / 2)}{\Gamma(n+b)}\left(\frac{\chi_{2(n+b), 1-\alpha}^{2}}{2}\right)^{1 / 2} \\
= & \sqrt{\frac{\pi}{4-\pi}}-\left(\sqrt{\frac{\pi}{4-\pi}}-1.246074685\right) \\
& \times \frac{\Gamma(5+1.001-1 / 2)}{\Gamma(5+1.001)}\left(\frac{\chi_{2(5+1.001), 0.95}^{2}}{2}\right)^{1 / 2} \\
= & 0.96984 .
\end{aligned}
$$


So, the $95 \%$ one-sided credible interval for $C_{L}$ is $\left[\underline{\mathrm{LB}}_{\mathrm{BS}}, \infty\right)=[0.96984, \infty)$.

$$
\begin{aligned}
\underline{\mathrm{LB}}_{\mathrm{BL}}= & \sqrt{\frac{\pi}{4-\pi}}-\left(\sqrt{\frac{\pi}{4-\pi}}-\widehat{C}_{L, \mathrm{BL}}\right) \\
& \times\left[\frac{1}{2 c}\left(1-e^{-c /(n+b+1)}\right) \chi_{2(n+b), 1-\alpha}^{2}\right]^{1 / 2} \\
= & \sqrt{\frac{\pi}{4-\pi}}-\left(\sqrt{\frac{\pi}{4-\pi}}-1.129561059\right) \\
& \times\left[\frac{1}{2(0.5)}\left(1-e^{-0.5 /(5+1.001+1)}\right) \chi_{2(5+1.001), 0.95}^{2}\right]^{1 / 2} \\
= & 0.96984 .
\end{aligned}
$$

So, the $95 \%$ one-sided credible interval for $C_{L}$ is $\left[\underline{\mathrm{LB}}_{\mathrm{BL}}, \infty\right)=[0.96984, \infty)$.

$$
\begin{aligned}
\underline{\mathrm{LB}}_{\mathrm{BG}}= & \sqrt{\frac{\pi}{4-\pi}}-\left(\sqrt{\frac{\pi}{4-\pi}}-\widehat{C}_{L, \mathrm{BG}}\right) \\
& \times\left[\frac{\Gamma(n+b+q / 2)}{\mathrm{T}(n+b)}\right]^{-1 / q}\left(\frac{\chi_{2(n+b), 1-\alpha}^{2}}{2}\right)^{1 / 2} \\
= & \sqrt{\frac{\pi}{4-\pi}}-\left(\sqrt{\frac{\pi}{4-\pi}}-1.200432998\right) \\
& \times\left[\frac{\Gamma(5+1.001+2 / 2)}{\Gamma(5+1.001)}\right]^{-1 / 2}\left(\frac{\chi_{2(5+1.001), 0.95}^{2}}{2}\right)^{1 / 2} \\
= & 0.96984 .
\end{aligned}
$$

So, the $95 \%$ one-sided credible interval for $C_{L}$ is $\left[\underline{\mathrm{LB}}_{\mathrm{BG}}, \infty\right)=[0.96984, \infty)$.

Step 5. Because of the performance index value $c_{0}=0.90 \notin$ $\left[\underline{\mathrm{LB}}_{\mathrm{BS}}, \infty\right)=\left[\underline{\mathrm{LB}}_{\mathrm{BL}}, \infty\right)=\left[\underline{\mathrm{LB}}_{\mathrm{BG}}, \infty\right)$, we reject the null hypothesis $H_{0}: C_{L} \leq 0.90$.

Thus, we can conclude that the lifetime performance index of data meets the required level. In addition, by using the prior parameter values $(a, b)=(6,1.5)$ based on the simulation studies, we also obtain that $c_{0}=0.90 \notin\left[\underline{\mathrm{LB}}_{\mathrm{BS}}, \infty\right)=$ $\left[\underline{\mathrm{LB}}_{\mathrm{BL}}, \infty\right)=\left[\underline{\mathrm{LB}}_{\mathrm{BG}}, \infty\right)=0.94033$. So, we also reject the null hypothesis $H_{0}: C_{L} \leq 0.90$. Hence, the lifetime performance index of data meets the required level.

\section{Conclusions}

Montgomery [1] proposed a process capability index $C_{L}$ for larger-the-better quality characteristic. The assumption of most process capability is normal distribution, but it is often invalid. In this paper, we consider that Rayleigh distribution is the special case of Weibull distribution.

Moreover, in lifetime data testing experiments, the experimenter may not always be in a position to observe the life times of all the products put to test. In industry and reliability studies, many products fail under stress, for example, an electronic component ceases to function in an environment of too high temperature, and a battery dies under the stress of time. So, in such experiments, measurement may be made sequentially and only the record values are observed.

In order to let the process capability, indices can be effectively used. This study constructs MLE and Bayesian estimator of $C_{L}$ under assuming the conjugate prior distribution and SE loss function, LINEX loss function, and GE loss function based on the upper record values from the Rayleigh distribution. The Bayesian estimator of $C_{L}$ is then utilized to develop a credible interval in the condition of known $L$.

This study also provides a table of the lifetime performance index with its corresponding conforming rate based on the Rayleigh distribution. That is, the conforming rate and the corresponding $C_{L}$ can be obtained.

Further, the Bayesian estimator and posterior distribution of $C_{L}$ are also utilized to construct the testing procedure of $C_{L}$ which is based on a credible interval. If you want to test whether the products meet the required level, you can utilize the proposed testing procedure which is easily applied and an effectively method to test in the condition of known $L$. Numerical example is illustrated to show that the proposed testing procedure is effectively evaluating whether the true performance index meets requirements.

In addition, these results from simulation studies illustrate that the performance of our proposed method is acceptable. According to SMSE, the Bayesian approach is smaller than the non-Bayesian approach, we suggest that the Bayesian approach is better than the non-Bayesian approach. Then, the SMSEs of prior parameters $(a, b)=(6,1.5)$ and $(2,5)$ are smaller than the SMSE of prior parameter $(a, b)=(2,2)$. We suggest that the prior parameters $(a, b)=(6,1.5)$ and $(2,5)$ are appropriate for the square-root inverted-gamma distribution based on the Bayesian estimators under the Rayleigh distribution.

\section{Acknowledgments}

The authors thank the referees for their suggestions and helpful comments on revising the paper. This research was partially supported by the National Science Council, China (Plans nos. NSC 101-2221-E-158-003, NSC 99-2118-M-415001, and NSC 100-2118-M-415-002). Moreover, J. W. Wu and C. W. Hong have a direct financial relation with the National Science Council, Taiwan, but other authors do not a direct financial relation with the National Science Council, Taiwan.

\section{References}

[1] D. C. Montgomery, Introduction To Statistical Quality Control, John Wiley \& Sons, New York, NY, USA, 1985.

[2] L. I. Tong, K. S. Chen, and H. T. Chen, "Statistical testing for assessing the performance of lifetime index of electronic components with exponential distribution," Journal of Quality Reliability Management, vol. 19, pp. 812-824, 2002.

[3] C.-W. Hong, J.-W. Wu, and C.-H. Cheng, "Computational procedure of performance assessment of lifetime index of 
businesses for the Pareto lifetime model with the right type II censored sample," Applied Mathematics and Computation, vol. 184, no. 2, pp. 336-350, 2007.

[4] C. W. Hong, J. W. Wu, and C. H. Cheng, "Computational procedure of performance assessment of lifetime index of Pareto lifetime businesses based on confidence interval," Applied Soft Computing, vol. 8, no. 1, pp. 698-705, 2008.

[5] J.-W. Wu, H.-M. Lee, and C.-L. Lei, "Computational testing algorithmic procedure of assessment for lifetime performance index of products with two-parameter exponential distribution," Applied Mathematics and Computation, vol. 190, no. 1, pp. 116-125, 2007.

[6] W.-C. Lee, J.-W. Wu, and C.-W. Hong, "Assessing the lifetime performance index of products with the exponential distribution under progressively type II right censored samples," Journal of Computational and Applied Mathematics, vol. 231, no. 2, pp. 648-656, 2009.

[7] W. C. Lee, J. W. Wu, and C. L. Lei, "Evaluating the lifetime performance index for the exponential lifetime products," Applied Mathematical Modelling, vol. 34, no. 5, pp. 1217-1224, 2010.

[8] K. N. Chandler, "The distribution and frequency of record values," Journal of the Royal Statistical Society B, vol. 14, pp. 220 228, 1952.

[9] S. K. Bhattacharya and R. K. Tyagi, "Bayesian survival analysis based on the rayleigh model," Trabajos de Estadistica, vol. 5, no. 1, pp. 81-92, 1990.

[10] A. D. Cliff and J. K. Ord, "Model building and the analysis of spatial pattern in human geography (with discussion)," Journal of the Royal Statistical Society B, vol. 37, no. 3, pp. 297-348, 1975.

[11] D. D. Dyer and C. W. Whisenand, "Best linear unbiased estimator of the parameter of the Rayleigh distribution. I. Small sample theory for censored order statistics," IEEE Transactions on Reliability, vol. R-22, no. 1, pp. 27-34, 1973.

[12] A. A. Soliman and F. M. Al-Aboud, "Bayesian inference using record values from Rayleigh model with application," European Journal of Operational Research, vol. 185, no. 2, pp. 659-672, 2008.

[13] StatSoft, STATISTICA Software, Version 7, StatSoft, Tulsa, Okla, 2005.

[14] W.-C. Lee, J.-W. Wu, M.-L. Hong, L.-S. Lin, and R.-L. Chan, "Assessing the lifetime performance index of Rayleigh products based on the Bayesian estimation under progressive type II right censored samples," Journal of Computational and Applied Mathematics, vol. 235, no. 6, pp. 1676-1688, 2011.

[15] M. Ahsanullah, Record Statistics, Nova Science, New York, NY, USA, 1995.

[16] B. C. Arnold, N. Balakrishnan, and H. N. Nagaraja, Records, John Wiley \& Sons, New York, NY, USA, 1998.

[17] P. W. Zehna, "Invariance of maximum likelihood estimators," Annals of Mathematical Statistics, vol. 37, p. 744, 1966.

[18] A. Zellner, "Bayesian estimation and prediction using asymmetric loss functions," Journal of the American Statistical Association, vol. 81, no. 394, pp. 446-451, 1986.

[19] S.-Y. Huang, "Empirical Bayes testing procedures in some nonexponential families using asymmetric linex loss function," Journal of Statistical Planning and Inference, vol. 46, no. 3, pp. 293-309, 1995.

[20] R. Calabria and G. Pulcini, "Point estimation under asymmetric loss functions for left-truncated exponential samples," Communications in Statistics-Theory and Methods, vol. 25, no. 3, pp. 585-600, 1996.
[21] A. T. K. Wan, G. Zou, and A. H. Lee, "Minimax and $\Gamma$-minimax estimation for the Poisson distribution under LINEX loss when the parameter space is restricted," Statistics and Probability Letters, vol. 50, no. 1, pp. 23-32, 2000.

[22] S. Anatolyev, "Kernel estimation under linear-exponential loss," Economics Letters, vol. 91, no. 1, pp. 39-43, 2006.

[23] X. Li, Y. Shi, J. Wei, and J. Chai, "Empirical Bayes estimators of reliability performances using LINEX loss under progressively type-II censored samples," Mathematics and Computers in Simulation, vol. 73, no. 5, pp. 320-326, 2007.

[24] D. K. Dyer and P. S. L. Liu, "On comparison of estimators in a generalized life model," Microelectronics Reliability, vol. 32, no. 1-2, pp. 207-221, 1992.

[25] A. A. Soliman, "Estimation of parameters of life from progressively censored data using Burr-XII model," IEEE Transactions on Reliability, vol. 54, no. 1, pp. 34-42, 2005.

[26] A. A. Soliman and G. R. Elkahlout, "Bayes estimation of the logistic distribution based on progressively censored samples," Journal of Applied Statistical Science, vol. 14, no. 3-4, pp. 281-293, 2005.

[27] C. Caroni, “The correct "ball bearings" data," Lifetime Data Analysis, vol. 8, no. 4, pp. 395-399, 2002.

[28] M. Z. Raqab and M. T. Madi, "Bayesian prediction of the total time on test using doubly censored Rayleigh data," Journal of Statistical Computation and Simulation, vol. 72, no. 10, pp. 781789, 2002.

[29] W. C. Lee, "Statistical testing for assessing lifetime performance Index of the Rayleigh lifetime products," Journal of the Chinese Institute of Industrial Engineers, vol. 25, pp. 433-445, 2008. 


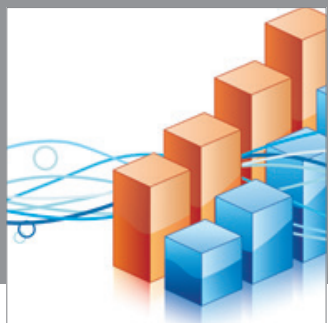

Advances in

Operations Research

mansans

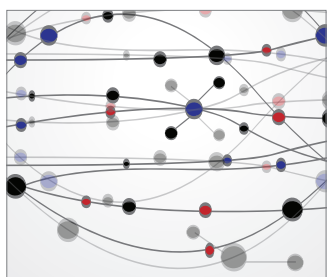

The Scientific World Journal
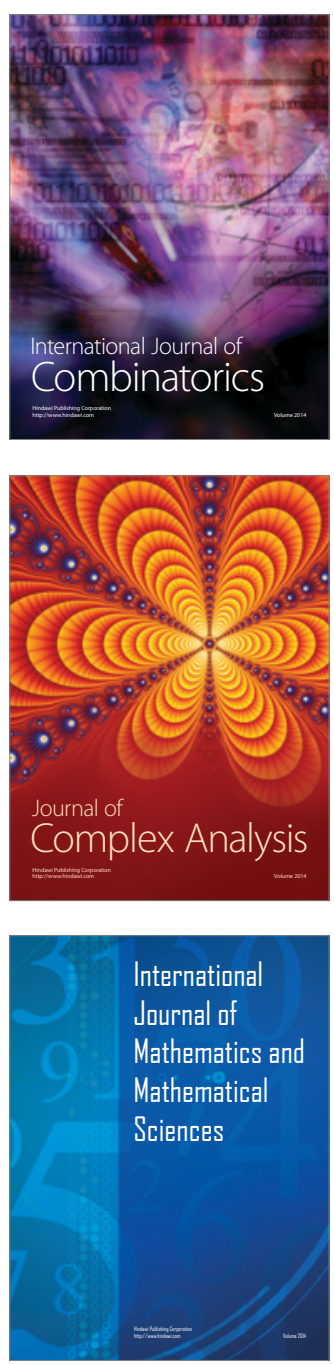
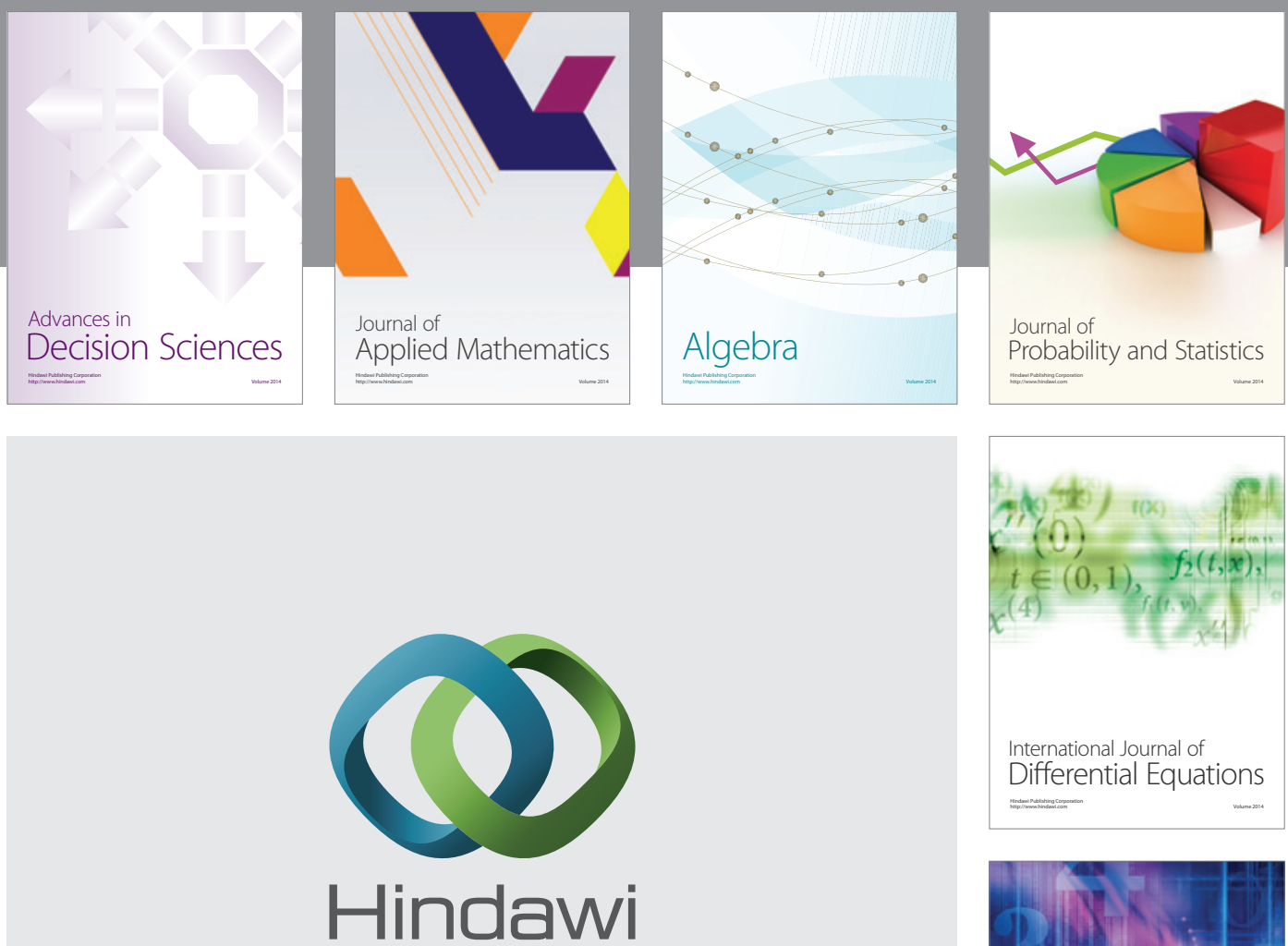

Submit your manuscripts at http://www.hindawi.com
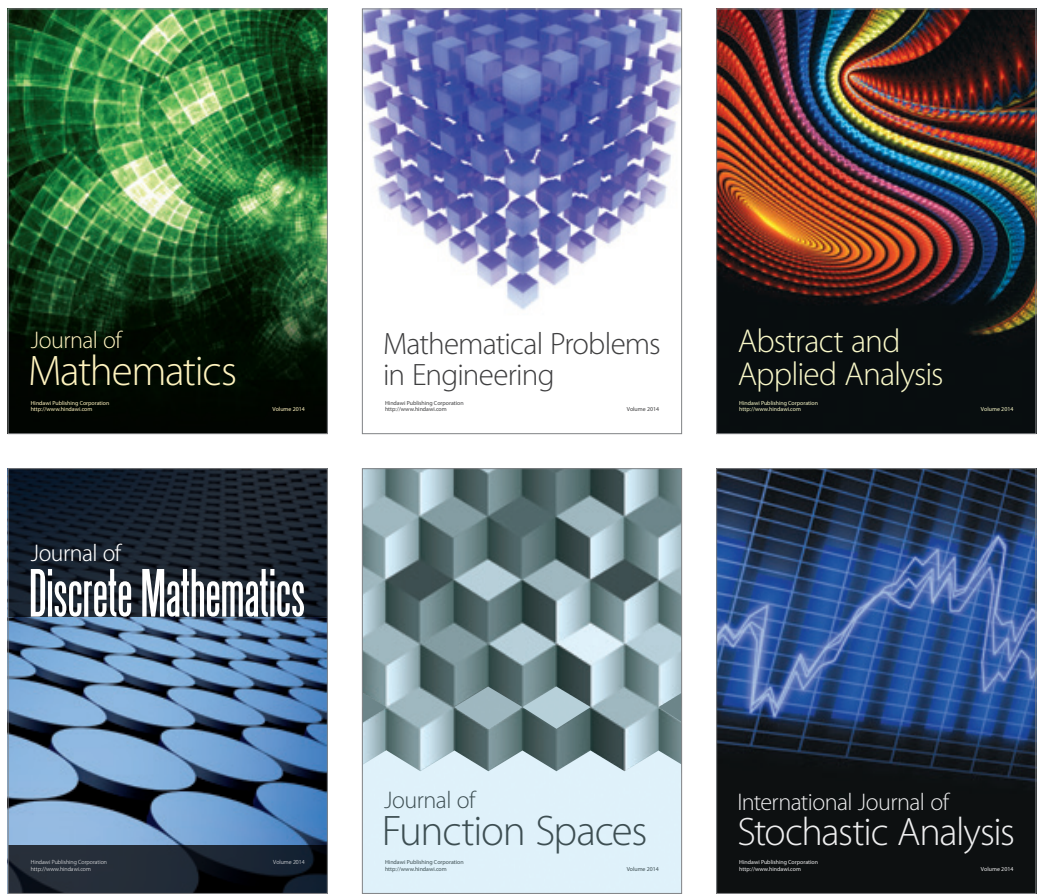

Journal of

Function Spaces

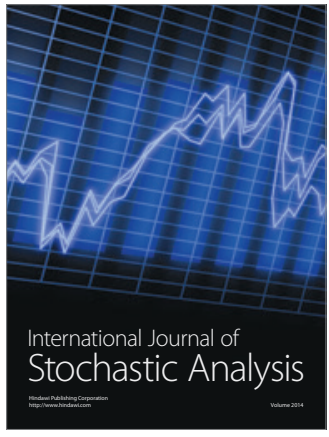

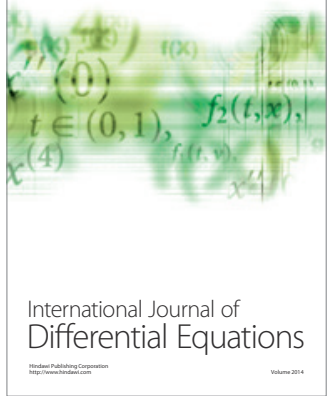
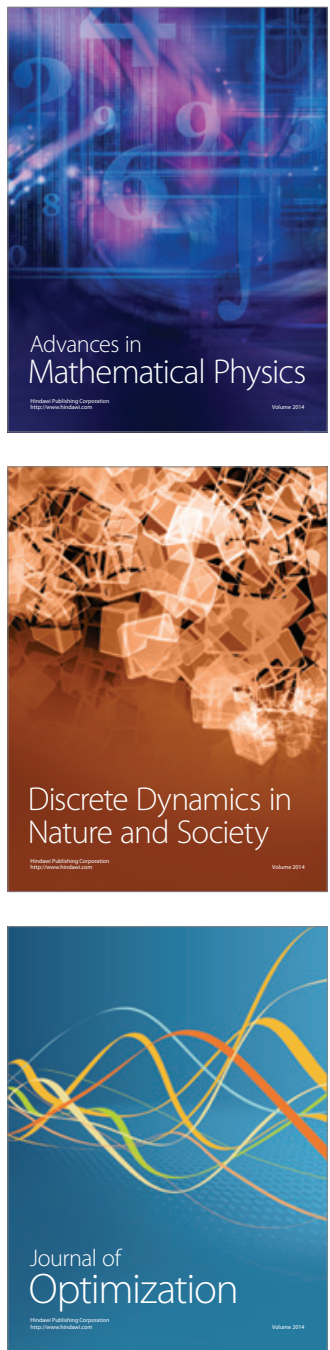